mechanisms in plants and simple forms of life; the author is mainly concerned with higher forms of life that are less able to adapt to harsh conditions.

The book is carefully crafted, well illustrated and largely devoid of typographical errors. The author even apologizes for using the letter $\mathrm{k}$ for the Boltzmann constant as well as a general-purpose coefficient; such consideration is rare. SI units are used throughout. Here is an original, amusing, well-produced and attractively priced book, ideal as a present to anyone with an ounce of natural curiosity about the fascinating interplay of physics and life on Earth.

Felix Franks is in the Biopreservation Division, Pafra Ltd, 150 Science Park, Cambridge CB4 4GG, UK.

\section{Random samples}

\section{Peter T. Landsberg}

\section{Physics and Chance: Philosophical Issues in the Foundations of Statistical Mechanics. By Lawrence Sklar. Cam- bridge University Press: 1993. Pp. 437. $£ 40, \$ 64.95$.}

THE tragedy of science is that we never know enough. That complete knowledge tends to escape us is nowhere more clearly illustrated than in statistical mechanics. As one cannot readily set up a system that follows the history of, say, $10^{23}$ particles, one has to eschew the search for completeness and substitute statistics. Statistical assumptions have to be made, probabilities enter and so on. As far as physics is concerned, it all started with Clerk Maxwell's distribution law in 1860 . The formalism of the new science of statistical mechanics grew and grew; it came to embrace solids, liquids and gases and now the subject has several physics journals devoted to it. Even the nuclear physics community makes space for papers on it.

What are these random elements? A good way to start discussing them is to design (on paper) a coin-tossing machine, such that if a coin is placed on it in a standard way, and a button is pressed, the coin is projected upwards and always comes down heads (say). If the design is bad, or the machine ages, it will become less reliable. Lack of knowledge and lack of control has then to be replaced by probabilities. Lawrence Sklar does not give this example, but he uses the first third of the book to tell readers about probabilities and the surprisingly many ways of approaching the relevant concepts. All these various avenues are in fact left open; the author is certainly evenhanded, but one would have liked some kind of summary for the sake of clarity.

In dealing with equilibrium and non- equilibrium systems (in the second third of the book), more advanced topics make their appearance, often without, I fear, adequate explanation: KAM theorem, BBGKY approach, Bernoulli systems, non-unitary transformations. Sklar is certainly a considerable scholar, but in this book perhaps not the ideal teacher. Most welcome, however, is his introduction of the spin-echo experiments of the 1950s. Here, it will be recalled, a certain order among atomic spins is destroyed by a radio-frequency pulse. The 'coarsegrained' appearance of the system is accordingly one of equilibrium and of higher entropy relative to the initial ordered state. But lo! Another pulse restores the status quo (that is, order). This shows rather well that there was lack of equilibrium in the system at all times and that a 'fine-grained' approach is essential. In such an approach the entropy remains constant throughout. Here again the author is perhaps not as emphatic as he might be.

There is also a revealing computer experiment (not mentioned in the book) in which particles interact, the entropy is calculated, and is found to increase with time. At a certain instant all velocities are reversed and the entropy decreases in the resulting spontaneous process. This follows Loschmid's request to Boltzmann in 1876 to see what happens to his $\mathrm{H}$ theorem in this case. Would he not find an entropy decrease? "You reverse them," was Boltzmann's answer (I believe), knowing that one could not reverse molecular velocities. As I say: now we can! We can also (on a computer) make a gas go into a corner of its container in an entropy-decreasing spontaneous process, by reversing the velocities that occur in the corresponding expansion. But the initial conditions for these processes are so incredibly delicate that the slightest computer errors (representing perhaps also a residual interaction with the outside world) tend to suppress this behaviour, and the system tends to go back to an entropy increase. It is the delicacy of the required initial conditions that explains (at least roughly) why an entropy decrease in an isolated system is never observed.

This takes us to the last third of the book, which deals with cosmology, the reduction of thermodynamics to statistical mechanics and time's arrow. Here a certain amount of speculation is inevitable. The author seems to favour the view that entropy continually increases in a re-contracting universe. (This depends on having at least two phases present.) There is a brief reference to arguments for the existence of God and for the author's view that entropy is not subjective, as well as some general remarks about the extension of concepts. The latter is illustrated by reference to the extension of the temperature concept to include negative temperatures.
This could have been made clearer by explicit reference to a system possessing only a finite energy spectrum.

The book occupies itself with foundations and touches on most of the crucial issues. It is, I believe, the only available modern text that has set itself this task, and as such it is recommended. While it cannot be expected to solve previously unsolved problems, it does not even classify them or bring them into sharp focus in a summary. This might be worth considering for a future edition.

The foundation problems are fairly profound; many remain unanswered, as the author points out in his conclusion, and a reader may come away with the idea that nothing very reliable can be erected on such sandy soil. This would of course be a grave error. The flow of electricity in solids, to give just one example, is often based on Boltzmann's transport equation, and is a going concern: the transport coefficients can be calculated and they often agree with experiment. While the exciting problems of the foundations of statistical mechanics is one of its glories, its widespread ability to reach out successfully to experiment is surely another which should be noted in a book of this kind.

Peter T. Landsberg is in the Department of Mathematics, University of Southampton, Southampton SO9 5NH, UK.

\section{New Journals issue}

This year, Nature's annual new journals review supplement will appear in the issue of 29 September. Publishers and learned societies are invited to submit journals for review, taking note of the following criteria:

Journals that first appeared during or after June 1992 and issued at least four separate numbers by the end of April 1994 will be considered

- Journals covering any aspect of science are eligible, although those dealing with clinical medicine, engineering and pure mathematics are excluded, as are publications of abstracts.

- Frequency of publication must be at least three times a year. The main language used must be English.

Translation journals in English are, of course, eligible.

Deadline for submission is the end of May.

When submitting journals for review, please send at least four different issues (the first, the most recent and any two others) of each title, together with full details of subscription rates. For further information please contact Peter Tallack, Nature, 4 Little Essex Street, London WC2R 3LF, 071-8366633 (011-44-71-836-6633 from the US), ext. 2414 